\title{
Distanciamento social: fôlego para ciência durante a pandemia de COVID-19 no Brasil
}

\section{Social distancing: breath for science during the COVID-19 pandemic in}

\section{Brazil}

Wellington Roberto Gomes de Carvalho'

Stefan Vilges de Oliveira ${ }^{2}$

Vivianne Peixoto da Silva ${ }^{1}$

Jean Ezequiel Limongi ${ }^{1}$

A COVID-19 é causada por um vírus respiratório (SARS-CoV-2), identificado pela primeira vez em Wuhan, província de Hubei, China em dezembro de 2019 [1-4]. Segundo os cientistas, os principais sintomas clínicos são febre, dor muscular, fadiga, tosse seca, dificuldade de respirar e evidência radiográfica do tórax de pneumonia $[2,5]$. Em resposta, as autoridades de saúde chinesas adotaram rigorosos procedimentos para rastrear e controlar a doença, incluindo medidas de isolamento de pessoas suspeitas da infecção, monitoramento, coleta de dados epidemiológicos e clínicos e a rápida realização de testes diagnóstico e tratamento de pacientes [2].

Em 31 de janeiro de 2020, na China haviam um total de 9.692 casos da COVID-195. Em 5 de maio de 2020, alguns meses depois do início da epidemia na China, já foram confirmados um total de 83.966 casos e 4.637 mortes [6]. Devido ao potencial de disseminação, a COVID-19 se espalhou rapidamente da China para outros países e continentes. Em todo o mundo foram registrados mais de 3,8 milhões de casos e 269 mil mortes6 e estimase que um número ainda maior será registrado nos meses subsequentes.

O Ministério da Saúde (MS) do Brasil em 27 de janeiro, ativou um plano de contingência com o objetivo de conter a entrada e a propagação da doença no território nacional [7]. Frente a urgência, a Secretaria de Vigilância em Saúde (SVS) do MS acionou o Centro de Operações de Emergência do MS para o planejamento, organização e monitoramento internacional [7]. Em 3 de fevereiro a epidemia é declarada Emergência em Saúde Pública de Importância Nacional [7] e em 26 de fevereiro de 2020 foi registrado o primeiro caso importado da COVID-19 no estado de São Paulo. Nos primeiros dias do mês de março, já haviam 488 casos suspeitos, 2 confirmados e 240 descartados no país, sem evidência ainda de transmissão local [8].

\footnotetext{
${ }^{1}$ Universidade Federal de Uberlândia, Instituto de Geografia, Uberlândia, MG, Brasil. E-mail: wrgcarvalho@ufu.br

${ }^{2}$ Universidade Federal de Uberlândia, Departamento de Saúde Coletiva, Uberlândia, MG, Brasil.
}

Como citar este artigo / How to cite this article

Carvalho WRG de et al. Distanciamento social: fôlego para ciência durante a pandemia de COVID-19 no Brasil Social distancing: breath for Science during the COVID-19 pandemic in Brazil. InterAm J Med Health 2020;3:e202003028. 
A crescente de casos e óbitos mostrou-se de forma exponencial no mês de abril e a crise se agrava em várias Unidades da Federação. O Brasil ultrapassa a China marco zero da doença, e chega a registrar em 8 de maio de 2020 um total de 135.773 casos confirmados e 9.190 mortes [6], ocupando o $8^{\circ}$ no ranking de infectados pelo coronavírus.

Na ausência de uma vacina e na tentativa de retardar a disseminação da COVID-19 a recomendação é tomar precauções [9]. Tendo em vista a falta de conhecimento científico sobre a COVID-19 [10] a capacidade de disseminação [11] e de causar óbitos [12] e a dúvida, acerca da melhor estratégia para controlar a pandemia, é fortemente sugerido o uso de medidas de contenção não farmacológicas, como as medidas de higiene/lavagem de mãos, uso de máscaras, distanciamento social e isolamento de casos suspeitos [13].

A pandemia avança e o Maranhão é o primeiro estado do país a aplicar medida radical "lockdown" nos quatro municípios da região metropolitana de São Luís. Com $100 \%$ dos leitos de unidade de terapia intensiva da rede pública de saúde na capital (São Luís) ocupados por pacientes infectados por COVID-19, a Vara de Interesses Difusos e Coletivos da Comarca da llha de São Luís ordenou, em 30 de abril, que o Maranhão e a capital adotem o bloqueio total das atividades, a partir do dia 05 de maio, por 10 dias. Outras cidades do país poderão colapso do sistema de saúde, como ocorrido no Maranhão, por aumento de casos da COVID-19. O cenário sugere que a medida que diminui a taxa de distanciamento social aumenta infecção [14].

A história mostra os perigos de relaxar restrições muito cedo em uma pandemia [15]. Um estudo que coletou dados históricos e epidemiológicos de mortalidade em grandes cidades dos EUA durante pandemia de gripe de 1918-1919, demostrou que pandemia de gripe de 115.340 mortes por pneumonia e influenza (taxa houve 115.340 mortes por pneumonia e influenza (taxa semanal de excesso de mortes (TSEM) de $500 / 100.000$ analisadas. Cada cidade adotou pelo menos uma das três principais categorias de intervenções não farmacológicas (fechamento de escola; cancelamento de reuniões públicas: e isolamento e quarentena). As restrições no funcionamento de escolas e funcionamento de escolas e reunies públicas alotadas simultaneamente representavam a combinaçáo mais
comum implementada em 34 cidades (79\%), e essa combinação teve uma duração mediana de quatro semanas sendo significativamente associada a reduções não farmacológicas apresentaram maiores atrosos atrasos matingr o pico de letalidade e as menores taxas de mortalidade. Houve uma associação significativa entre o aumento da duração das intervenções não farmacológicas e da redução da carga total de mortalidade [15].

A estratégia de manter o maior número de pessoas em casa, ajudou a salvar vidas, deu mais tempo para os cientistas buscar tratamentos e desenvolverem vacinas, e para as autoridades de saúde pública e o sistema de saúde planejar a logística, testar e tratar pacientes. Isto contribuiu para o achatamento da curva.

Neste sentido, é exatamente o que todos desejam. Frente a pandemia, a recomendação de distanciamento social é um esforço para o achatar a curva e retardar a propagação do coronavírus, reduzir o número de casos e de óbitos, e descobrir medicamentos e vacina eficiente. Precisamos manter o curso, evitar mais mortes e ganhar mais tempo para a ciência encontrar a solução. É melhor prevenir do que remediar.

\section{REFERÊNCIAS}

Wang Chen, Horby Peter W, Hayden Frederick $G$ Gao George $F$. A novel coronavirus outbreak of globa health concern. The Lancet 2020:395(10223):470473. doi.org/10.1016/50140-6736(20)30185-9

2. World Health Organization. Pneumonia of unknown cause - China: disease outbreak news. Geneva: World Health Organization. 2020 (accessed 2020 Apr 30). Available at: https://umwwho int/crr/don/05-january2020-pneumonia-of-unkown-cause-china/en/

3. Lu H, Stratton CW, Tang YW. Outbreak of pneumoni of unknown etiology in Wuhan China: the mystery and the miracle. J Med Virol. 2020;92:401-402. (accessed 2020 May 02). doi:10.1002/jmv.25678

4. Paules $\mathrm{Cl}$, Marston HD, Fauci AS. Coronavirus infections-more than just the common cold. JAMA. 2020;323(8):707-708. doi:10.1001/jama.2020.0757
5. Wang D, Hu B, Hu C, Zhu F, Liu X, Zhang J. et al. Clinical Characteristics of 138 Hospitalized Patients With 2019 Novel Coronavirus-Infected Pnemonta in Wuhan, China. 2020:323(11):1061冈1069. doi:10.1001/jama. 2020.1585

6. Johns Hopkins University. Coronavirus COVID-19 Global Cases by Johns Hopkins CSSE [Internet] Johns Hopkins University; 2020 (accessed 2020 May 08] Available at: https://gisanddata.maps. arcgis. com/apps/opsdashboard/index htmlt/ bda7594740fd40299423467b48e9ecf6

7. Croda JHR, Garcia LP. Resposta imediata da Vigilância em Saúde à epidemia da COVID-19. Epidemiol. Sen Saúde 2020;29(1):e2020002. doi: 10.5123/S167949742020000100021

8. Brasil. Ministério da Saúde. Portaria MS/GM n 188 , de 3 de fevereiro de 2020. Declara Emergência em Saúde Pública de importância Nacional(ESPIN) em decorrência da Infecção Humana pelo novo Coronavírus (2019nCoV) [Internet]. Diário Oficial da União, Brasilia (DF), 2020 fev 4;Seção Extra:1. (acessado $2020 \mathrm{Ma}$ 02). Disponível em: http://portalarquivos.saude.gov. br/images/pdf/2020/April/06/2020-04-06---BE7--Boletim-Especial-do-COE---Atualizacao-da-Avaliacaode-Risco.pdf

9. Centers for Disease Control and Prevention Coronavirus Disease 2019 (COVID-19). How to Protect Yourself and Others. 2019 (accessed 2020 May 01). Available at:https://www.cdc.gov/coronavirus/2019ncov/index.html/

10. Anderson RM, Heesterbeek $H$, Klinkenberg $D$ Hollingsworth TD. How will country-based mitigation measures measures influence the course of the COVID-19 epidemic? Lancet 2020; 395(10228): 931-4 do org/10.1016/50140-6736(20)30567-5

11. Liu Y, Gayle AA, Wilder-Smith A, Rocklöv J. The reproductive number of COVID-19 is higher compared to SARS coronavirus. J Travel Med 2020; 27(2): 1-4. doi.org/10.1093/tm/taaa021

12. The Novel Coronavirus Pneumonia Emergency Response Epidemiology Team. The Epidemiologica
Characteristics of an Outbreak of 2019 Novel Coronavirus Diseases (COVID-19) - China, 2020[J]. China CDC Weekly. 2020;2(8):113-122. (accessed 2020 May 03). Available at: http://weekly.chinacdc. cn/en/article/id/e53946e2-c6c4-41e9-9a9bfea8db1a8f51

13. Brasil. Ministério da Saúde. Boletim COE COVID-19 Centro de Operações de Emergência em Saúde Pública. Doença pelo Coronavírus 2019. n 12, de 19 de abril de 2020. Especial Vigilância Epidemiológica Labortal 2020; Semana Epideniónica: 17 (192). Disponível em:http:// 25/04). (acessado 2020 Mai 02). Disponivel em:http:// portalarquivos.saude.gov.br/images/pdf/2020/ April/19/BE12-Boletim-do-COE.pdf

14. Ganem F, Mendes FM, Oliveira SB, Porto VBG, Araujo $\mathrm{W}$, Nakaya $\mathrm{H}$ et al. The impact of early social distancing at COVID-19 Outbreak in the largest Metropolitan Area of Brazil. medRxiv. 2020; doi. org/10.1101/2020.04.06.20055103

15. Markel H, Lipman HB, Navarro JA, Sloan UM, Michalsen $J R$, Stern AM et al. Nonpharmaceutical Interventions Implemented by US Cities During the 1918-1919 Influenza Pandemic. JAMA. 2007;298(6):644-654. (accessed 2020 May 02). Available at: doi:10.1001/ jama.298.6.644

Telefone: (34) 3230-9432 